\title{
Adiciones a la pteridoflora de Tabasco, México: la importancia del bosque mesófilo de montaña
}

\section{Additions to the pteridoflora of Tabasco, Mexico: the importance of the humid montane forest}

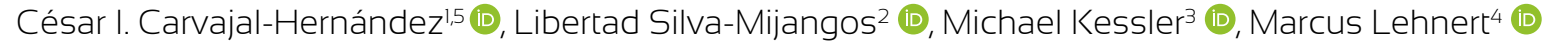

1 Universidad Veracruzana, Instituto de Investigaciones Biológicas, Av. Dr. Luis Castelazo Ayala s/n, Col. Industrial Ánimas, 91190 Xalapa, Veracruz, México.

2 Universidad de Ciencias y Artes de Chiapas, sede Mapastepec, Libramiento norte $\mathrm{s} / \mathrm{h}$, barrio Luis Donaldo Colosio, 30506 Mapastepec, Chiapas, México.

3 University of Zurich, Institute of Systematic and Evolutionary Botany, Zollikerstrasse 107, 8008 Zurich, Suiza.

4 University of Bonn, Nees Institute for Biodiversity of Plants, Meckenheimer Allee 170, D-53115 Bonn, Alemania.

5 Autor para la correspondencia: ccarvajal@uv.mx

Recibido: 13 de octubre de 2017 Revisado: 31 de octubre de 2017. Aceptado: 23 de noviembre de 2017. Primero en línea: 29 de mayo de 2018. Publicado: 1 de julio de 2018.

\section{Citar como:}

Carvajal-Hernández, C. I., L. Silva-Mijangos, M. Kessler y M. Lehnert. 2018. Adiciones a la pteridoflora de Tabasco, México: la importancia del bosque mesófilo de montaña. Acta Botanica Mexicana 124: 7-18. DOI: 10.21829/abml24.2018.1300

DOI:

$10.21829 / \mathrm{abml} 24.2018 .1300$

\section{RESUMEN:}

Antecedentes y Objetivos: El ejido Villa de Guadalupe del municipio Huimanguillo, Tabasco, mantiene el único fragmento de bosque mesófilo de montaña en el estado, mismo que ha sido florísticamente poco explorado. Este estudio se suma a otros anteriormente publicados, donde los reportes de nuevos registros aumentan considerablemente la riqueza de especies de helechos y licopodios conocidas hasta ahora para Tabasco. El objetivo principal es actualizar el conocimiento de la pteridoflora de la entidad, enfatizando la importancia del bosque mesófilo de montaña.

Métodos: Se realizaron colectas al azar de helechos epífitos y terrestres en la cara norte y noreste del cerro Las Flores (450-1000 m s.n.m.) en el municipio Huimanguillo. Los especímenes fueron identificados principalmente mediante claves taxonómicas y distribuidos en herbarios nacionales y extranjeros.

Resultados clave: Se registraron en total 64 especies de helechos y licopodios. Las familias mejor representadas fueron Polypodiaceae, Dryopteridaceae e Hymenophyllaceae. Se destaca el registro de 30 especies que no habían sido reportadas previamente en el estado de Tabasco. Para los géneros Cochlidium, Elaphoglossum, Hypolepis, Odontosoria, Parapolystichum, Polytaenium, Saccoloma y Sphaeropteris, este es el primer reporte para la entidad.

Conclusiones: Las nuevas adiciones a la pteridoflora de Tabasco proporcionan un recuento de 169 especies para el estado, lo cual actualiza la información conocida hasta ahora (137 especies) y manifiesta la necesidad de continuar con la realización de inventarios florísticos regionales. El presente estudio representa uno de los pocos trabajos publicados que documentan la riqueza de un grupo taxonómico en específico en el bosque mesófilo de montaña del estado.

Palabras clave: helechos, Huimanguillo, inventario florístico, licopodios, nuevos registros, Pteridofitas.

\section{ABSTRACT:}

Background and Aims: The ejido Villa de Guadalupe of the municipality of Huimanguillo, Tabasco, contains the only fragment of humid montane forest in the state, which has been little explored floristically. This study is an addition to those previously published, where reports of new records increase considerably the richness of fern species known so far for Tabasco state. The main objective is to update the knowledge of the pteridophyte flora of this state, emphasizing the importance of the humid montane forest.

Methods: Epiphytic and terrestrial fern collections were made randomly on the north and northeast face of the Las Flores mountain (450-1000 m a.s.1.) in the municipality of Huimanguillo. Specimens were identified mainly by dichotomous keys and distributed to national and foreign herbaria.

Key results: A total of 64 species of ferns and lycopods were recorded. The best represented families were Polypodiaceae, Dryopteridaceae and Hymenophyllaceae. We highlight the records of 30 species that had not previously been reported in the state of Tabasco. Even of the genera Cochlidium, Elaphoglossum, Hypolepis, Odontosoria, Parapolystichum, Polytaenium, Saccoloma and Sphaeropteris, this is the first report for the state.

Conclusions: The new additions to the pteridophyte flora of Tabasco lead to a count of 169 species for the state, which updates the information known so far (137 species) and shows the need of continued regional floristic inventories. The present study represents one of the few published papers that document the richness of a specific taxonomic group in the humid montane forest of the state.

Key words: ferns, floristic inventory, Huimanguillo, lycopods, new records, pteridophytes. 


\section{INTRODUCCIÓN}

La pteridoflora de México es una de las que presenta mayor diversidad a nivel mundial. Se reporta que existen entre 1030 y 1039 especies (Tejero-Díez et al., 2014; Villaseñor, 2016), lo que representa cerca de $10 \%$ de la diversidad de helechos en el mundo (Mickel y Smith, 2004), aunque registros recientes siguen incrementando este número (p. ej. Rojas-Alvarado y Tejero-Díez, 2017; Sundue, 2017). Esto confirma también que la pteridoflora mexicana es una de las que mayor estudio han tenido en el país a lo largo de la historia. Así lo demuestran publicaciones de estudios regionales y/o estatales en el norte de México (Knobloch y Correll, 1962), en la región centro-occidente (Díaz-Barriga y PalaciosRíos, 1992; Mickel, 1992; Riba et al., 1996; ArreguínSánchez et al., 2001; Tejero-Díez y Arreguín-Sánchez, 2004) y en el sur del país (Smith, 1981; Mickel y Beitel, 1988; Magaña-Alejandro, 1992; Palacios-Ríos, 1992), hasta la obra The Pteridophytes of Mexico de Mickel y Smith (2004). Recientemente se publicaron trabajos como los helechos del estado de Hidalgo (SánchezGonzález et al., 2017) y las especies de helechos epífitos de Veracruz (Mendoza-Ruíz et al., 2016), por citar algunos ejemplos.

La investigación florística previa demuestra que los estados con mayor diversidad de helechos y licopodios en México son Chiapas (697 especies), Oaxaca (667) y Veracruz (564) (Mickel y Smith, 2004; Krömer et al., 2015). Sin embargo, aún quedan estados o regiones que requieren de estudios más detallados. Un ejemplo de ello lo representa el estado de Tabasco, en el cual se han realizado pocos estudios que documenten la riqueza de especies de helechos y licopodios. De los trabajos que tratan estos grupos de plantas, el de Cowan (1983) reporta 84 especies y Magaña-Alejandro (1992) registra la presencia de 118. La misma cantidad es mencionada por Mickel y Smith (2004), aunque con especies distintas; incluso los autores no incluyen algunos taxa que sí son considerados por Magaña-Alejandro (1992), por no haber sido verificados. Por otro lado, Pérez et al. (2005), basados en un estudio de campo focalizado en zonas específicas del estado, reportan únicamente 85 especies en el apartado de flora de la obra Biodiversidad de Tabasco (Cuadro 1).

Cuadro 1: Comparación de números de familias, géneros y especies de helechos y licopodios registrados para el estado de Tabasco en diferentes estudios. Datos no disponibles $=\mathrm{n} / \mathrm{d}$.

\begin{tabular}{lccc}
\hline Autor & Familias & Géneros & Especies \\
\hline Cowan (1983) & 20 & 37 & 84 \\
Magaña-Alejandro (1992) & 21 & 40 & 118 \\
Mickel y Smith (2004) & 25 & 46 & 118 \\
Pérez et al. (2005) & 16 & 35 & 85 \\
Cetzal-Ix et al. (2013a, b) & $\mathrm{n} / \mathrm{d}$ & $\mathrm{n} / \mathrm{d}$ & 137 \\
Este estudio & 25 & 54 & 169 \\
\hline
\end{tabular}

La realización de inventarios florísticos, en conjunto con la revisión de ejemplares de herbario (Bebber et al., 2010), en algunas regiones de México, sobre todo donde la información es escasa y con grupos taxonómicos específicos, sigue siendo una tarea vigente y necesaria (Magaña y Villaseñor, 2002; Krömer et al., 2015; Vergara-Rodríguez et al., 2017). Para el caso concreto del estado de Tabasco, han transcurrido más de 20 años desde el estudio de Magaña-Alejandro (1992), para que se registraran 21 nuevas especies para la pteridoflora de ese estado, basado en trabajo de campo y herbario (Cetzal-Ix et al., 2013a, b).

En el presente trabajo, se muestran los resultados de las recolectas de helechos y licopodios realizadas en el ejido Villa de Guadalupe del municipio Huimanguillo. La zona mantiene el único fragmento de bosque mesófilo de montaña en el estado de Tabasco, y son escasos los estudios florísticos publicados previamente de este tipo de vegetación. Por lo tanto, este estudio tiene como objetivo contribuir a la actualización del listado de especies de helechos y licopodios conocidos hasta ahora en Tabasco, destacando la importancia que tiene el bosque mesófilo de montaña como zona de resguardo para estos grupos taxonómicos. 


\section{Materiales y Métodos}

\section{Área de estudio}

El estado de Tabasco se localiza al sureste de México en la costa del Golfo de México y presenta un relieve plano en la mayor parte de su territorio. Pequeñas porciones al sur están cubiertas por sierras que alcanzan altitudes de 500 a 1000 m en las zonas limítrofes con los estados de Veracruz y Chiapas, así como en la frontera con Guatemala (Díaz-Jiménez et al., 2015). El muestreo fue realizado en la cara norte y noreste de la localidad conocida como cerro Las Flores, perteneciente al ejido Villa Guadalupe (región Agua Selva), en el municipio Huimanguillo, cerca de las colindancias con los estados de Chiapas y Veracruz (Fig. 1). La zona de estudio contiene en su superficie un fragmento de bosque mesófilo de montaña en buen estado de conservación que ocupa una franja de cerca de $300 \mathrm{~m}$ de altitud, ya que se distribuye desde los 700 hasta los 1000 m s.n.m., abarcando una superficie aproximada de $0.9 \mathrm{~km}^{2}$. En un rango altitudinal de 500 a $700 \mathrm{~m}$, se encuentra representada una zona de transición entre la selva mediana subperennifolia y bosque mesófilo de montaña. Por debajo de $500 \mathrm{~m}$, cercano a las comunidades Francisco J. Mújica y Villa de Guadalupe, predominan fragmentos de selva mediana subperennifolia rodeados de fracciones de terreno ocupadas por vegetación secundaria.
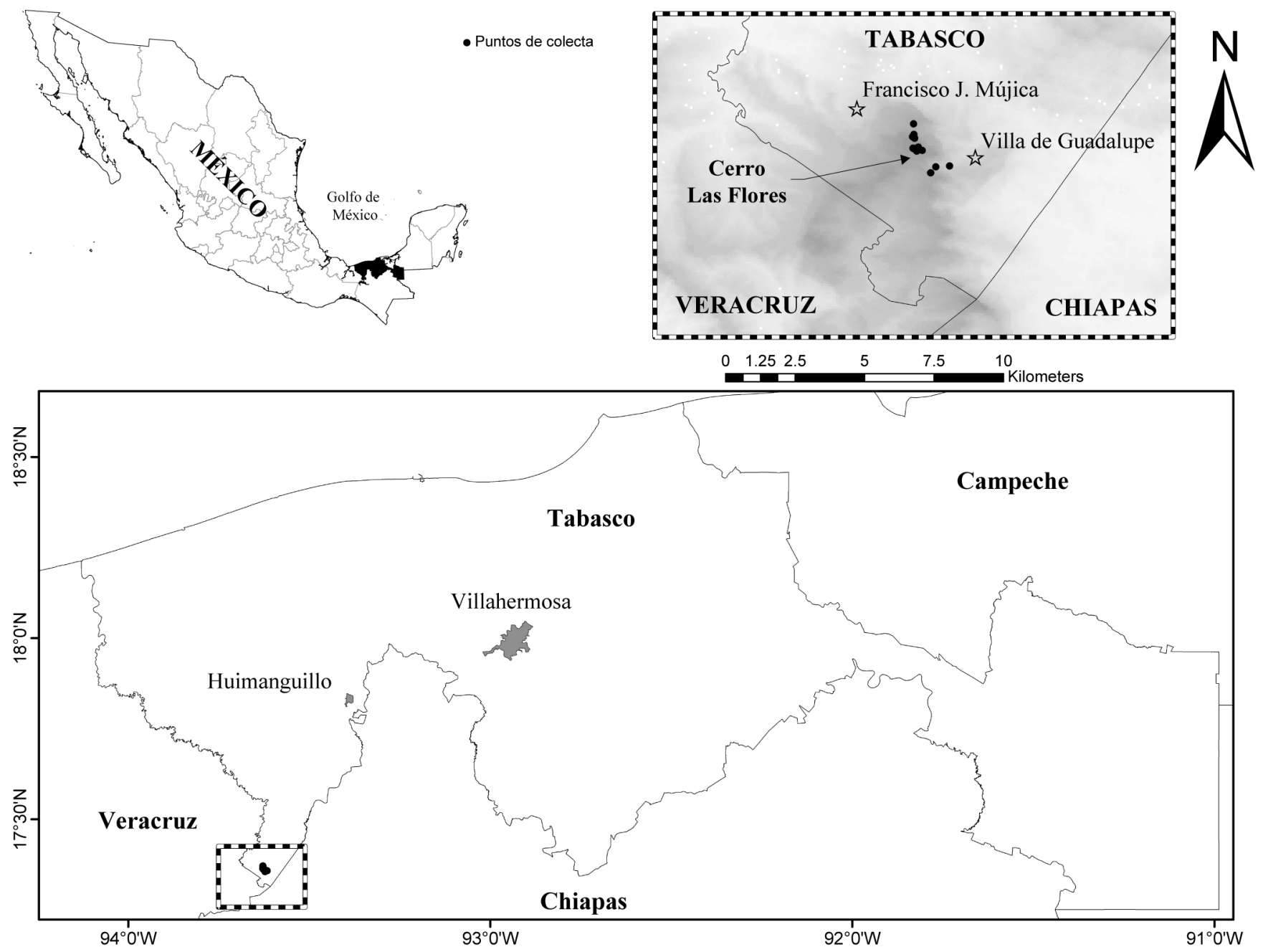

Figura 1: Ubicación de los puntos de colecta de helechos y licopodios en el cerro Las Flores del ejido Villa de Guadalupe, Huimanguillo, Tabasco, México. 
El clima en la mayor parte del estado es de tipo cálido húmedo con lluvias abundantes en verano (Salazar, 1994). Presenta una temperatura media anual de 26 ${ }^{\circ} \mathrm{C}$, mientras que la precipitación promedio anual es entre $1500 \mathrm{~mm}$ en la costa y $3000 \mathrm{~mm}$ en la zona serrana (INEGI, 1999). No existe información de los aspectos climáticos en la zona de estudio ya que las estaciones cercanas se encuentran a 100 m s.n.m.; por lo tanto, los valores que registran no corresponden a las altitudes como las que presenta este sitio. En consecuencia, se consultó la información climática disponible en "Climatologies at high resolution for the earth's land surface áreas (CHELSA)", la cual es una base de datos con información del clima a nivel mundial que considera las diferencias en temperatura y precipitación en distintas altitudes (Karger et al., 2017). Con base en lo anterior, la zona de estudio presenta una temperatura media anual de $20.6{ }^{\circ} \mathrm{C}$ y una precipitación anual de $3638 \mathrm{~mm}$.

\section{Trabajo de campo}

Se realizaron recolectas intensivas de helechos y licopodios en diferentes altitudes del ejido Villa de Guadalupe (450, 600, 800 y 1000 m s.n.m.), las cuales se hicieron al azar tanto en la cara norte como noreste del cerro Las Flores, ingresando por las comunidades Francisco J. Mújica y Villa de Guadalupe respectivamente. Los tipos de vegetación muestreados fueron la selva mediana subperennifolia, bosque mesófilo de montaña y la zona de transición entre ambos tipos de vegetación. Se colectaron especímenes tanto terrestres como epífitos (altura aproximada de cinco metros o epífitos del dosel que se encontraron en ramas caídas). La colecta de muestras botánicas se realizó de acuerdo con las técnicas propuestas en Lot y Chiang (1986).

\section{Determinación de especies}

Los ejemplares fueron determinados con base en la obra de Mickel y Smith (2004) y consulta a especialistas (ver agradecimientos). La clasificación taxonómica se realizó de acuerdo con Mickel y Smith (2004) y Smith et al. (2006). Las actualizaciones nomenclaturales se uni- formizaron según Ranker et al. (2004), Ebihara et al. (2006), Moran et al. (2010); Kessler et al. (2011a), Lehnert (2012), Smith y Tejero-Díez (2014) y Oliveira et al. (2017). Para el ordenamiento de las familias fue utilizada la clasificación de The Pteridophyte Phylogeny Group (PPG I, 2016).

Las recolectas se depositaron en el herbario CIB del Instituto de Investigaciones Biológicas, Universidad Veracruzana. Los duplicados han sido distribuidos parcialmente al Herbario Nacional en el Instituto de Biología de la Universidad Nacional Autónoma de México (MEXU) y al Herbario de la Universidad de CaliforniaBerkeley (UC).

\section{Resultados}

Se contabilizaron 64 especies (tres licopodios, 61 helechos) distribuidas en 41 géneros (Cuadro 2). Las familias mejor representadas fueron Polypodiaceae (14 especies), Dryopteridaceae (8) e Hymenophyllaceae (6). Se destaca que 30 especies no habían sido reportadas previamente en el estado de Tabasco. Incluso, para los géneros Cochlidium Kaulf., Elaphoglossum Schott ex J. Sm., Hypolepis Bernh., Lastreopsis Ching, Odontosoria Fée, Polytaenium Desv., Saccoloma Kaulf., y Sphaeropteris Bernh., este es el primer registro para la entidad.

Del total de especies consideradas, 33 fueron terrestres, 29 epífitas y dos hemiepífitas. El bosque mesófilo fue el ecosistema con mayor riqueza de especies, ya que alberga $78 \%$ de la riqueza total (50 especies) en una franja altitudinal desde 700 hasta $1000 \mathrm{~m}$. Las otras 14 especies se encuentran por debajo de los $700 \mathrm{~m}$ s.n.m. en la zona de transición entre el bosque mesófilo de montaña y la selva mediana subperennifolia.

\section{DISCUSIÓN}

En la pteridoflora de Tabasco se habían registrado 137 especies (Magaña-Alejandro, 1992; Mickel y Smith, 2004; Cetzal-Ix et al., 2013a, b). Este número está basado en el trabajo de Cetzal-Ix (2013a) donde se menciona que Magaña-Alejandro (1992) considera 116 especies para el estado. Sin embargo, en dicha obra en realidad son repor- 
Cuadro 2: Listado de especies de helechos y licopodios registrados en el cerro Las Flores, ejido Villa de Guadalupe, Huimanguillo, Tabasco. Las muestras se encuentran depositadas en el herbario CIB (Universidad Veracruzana), con duplicados en UC (Universidad de California-Berkeley) y MEXU (Universidad Nacional Autónoma de México). Entre paréntesis se indican los nombres abreviados de los colectores (CICH=César I. Carvajal-Hernández, LSM=Libertad Silva Mijangos) y sus números de colecta. Se indican el Hábito (H): Terrestre (T), Epífito (E), Hemiepífito (HE) y el tipo de vegetación (Veg): bosque mesófilo de montaña (B), selva mediana subperennifolia (s), zona de transición (t). Las especies en negritas corresponden a los nuevos registros para el estado. *Ejemplares sin identificar a nivel especifico debido a que se requiere mayores estudios para corroborar que se trata de especies o híbridos sin describir.

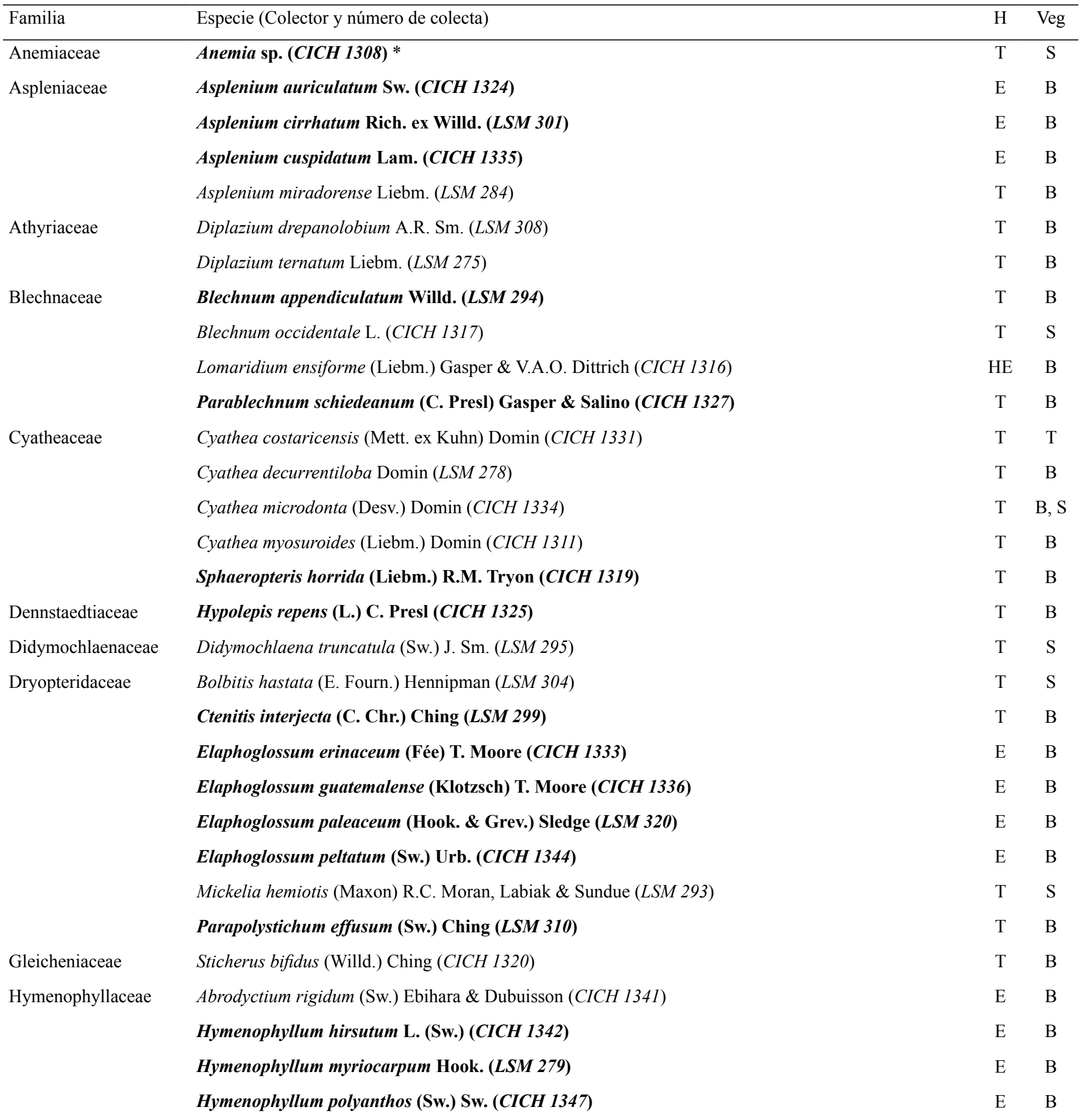


Cuadro 2: Continuación.

\begin{tabular}{|c|c|c|c|}
\hline Familia & Especie (Colector y número de colecta) & $\mathrm{H}$ & Veg \\
\hline & Polyphlebium capillaceum (L.) Ebihara \& Dubuisson (CICH 1339) & $\mathrm{E}$ & B \\
\hline & Vandenboschia collariata (Bosch) Ebihara \& K. Iwats. (LSM 298) & $\mathrm{E}$ & $\mathrm{B}$ \\
\hline Lindsaeaceae & Odontosoria schlechtendalii (C. Presl) C. Chr. (CICH 1315) & $\mathrm{T}$ & $\mathrm{B}$ \\
\hline Lomariopsidaceae & Lomariopsis mexicana Holttum (LSM 296) & HE & $\mathrm{B}$ \\
\hline Lycopodiaceae & Lycopodium cernuum L. (CICH 1321) & $\mathrm{T}$ & $\mathrm{B}$ \\
\hline \multirow[t]{14}{*}{ Polypodiaceae } & Campyloneurum xalapense Fée (CICH 1310) & $\mathrm{E}$ & $\mathrm{B}$ \\
\hline & Cochlidium linearifolium (Desv.) Maxon ex C. Chr. (CICH 1338) & $\mathrm{E}$ & $\mathrm{B}$ \\
\hline & Cochlidium serrulatum (Sw.) L.E. Bishop (CICH 1340) & $\mathrm{E}$ & $\mathrm{B}$ \\
\hline & Moranopteris taenifolia (Jenman) R.Y. Hirai \& J. Prado (CICH 1332) & $\mathrm{E}$ & $\mathrm{B}$ \\
\hline & Pecluma alfredii (Rosenst.) M.G. Price (LSM 277) & $\mathrm{E}$ & $\mathrm{B}$ \\
\hline & Pecluma sursumcurrens (Copel.) M.G. Price (CICH 1328) & $\mathrm{E}$ & $\mathrm{B}$ \\
\hline & Pleopeltis angusta Humb. \& Bonpl. ex Willd. (CICH 1346) & $\mathrm{E}$ & $\mathrm{B}$ \\
\hline & Pleopeltis astrolepis (Liebm.) E. Fourn. (LSM 306) & $\mathrm{E}$ & B \\
\hline & Pleopeltis collinsii (Maxon) A.R. Sm. \& Tejero (CICH 1330) & $\mathrm{E}$ & $\mathrm{B}$ \\
\hline & Pleopeltis polypodioides (L.) E.G. Andrews \& Windham (LSM 307) & $\mathrm{E}$ & $\mathrm{B}$ \\
\hline & Pleopeltis sp. (CICH 1329)* & $\mathrm{E}$ & $\mathrm{B}$ \\
\hline & Polypodium echinolepis Fée (CICH 1326) & $\mathrm{E}$ & $\mathrm{B}$ \\
\hline & Polypodium rhachipterygium Liebm. (LSM 321) & $\mathrm{E}$ & $\mathrm{B}$ \\
\hline & Serpocaulon triseriale (Sw.) A.R. Sm. (LSM 280) & $\mathrm{E}$ & $\mathrm{B}$ \\
\hline \multirow[t]{5}{*}{ Pteridaceae } & Adiantum wilesianum Hook. (LSM 305) & $\mathrm{T}$ & $\mathrm{S}$ \\
\hline & Polytaenium feei (W. Schaffn. ex Fée) Maxon (LSM 303) & $\mathrm{E}$ & B \\
\hline & Pteris altissima Poir. (LSM 286) & $\mathrm{T}$ & $\mathrm{S}$ \\
\hline & Pteris quadriaurita Retz. (CICH 1309) & $\mathrm{T}$ & $\mathrm{B}$ \\
\hline & Vittaria lineata (L.) Sm. (CICH 1337) & $\mathrm{E}$ & B \\
\hline Saccolomataceae & Saccoloma inaequale (Kunze) Mett. (LSM 292) & $\mathrm{T}$ & $\mathrm{B}$ \\
\hline \multirow[t]{2}{*}{ Selaginellaceae } & Selaginella finitima Mickel \& Beitel (CICH 1318) & $\mathrm{T}$ & $\mathrm{T}$ \\
\hline & Selaginella martensii Spring (LSM 289) & $\mathrm{T}$ & B \\
\hline Tectariaceae & Tectaria incisa Cav. (LSM 302) & $\mathrm{T}$ & $\mathrm{S}$ \\
\hline \multirow[t]{5}{*}{ Thelypteridaceae } & Amauropelta oligocarpa (Humb. \& Bonpl. ex Willd.) Pic. Serm (CICH 1312) & $\mathrm{T}$ & $\mathrm{T}$ \\
\hline & Christella dentata (Forssk.) Brownsey \& Jermy (CICH 1313) & $\mathrm{T}$ & $\mathrm{T}$ \\
\hline & Goniopteris hatchii (A.R. Sm.) Á. \& D. Löve (LSM 276) & $\mathrm{T}$ & $\mathrm{B}$ \\
\hline & Goniopteris hondurensis (L.D. Gómez) Salino \& T.E. Almeida (CICH 1323) & $\mathrm{T}$ & $\mathrm{S}$ \\
\hline & Macrothelypteris torresiana (Gaudich.) Ching (CICH 1314) & $\mathrm{T}$ & $\mathrm{S}$ \\
\hline
\end{tabular}


tadas 118. Esta misma cantidad concuerda con la cifra de Mickel y Smith (2004), aunque algunas de las especies no coinciden en ambos trabajos debido a que en la obra más antigua no fueron verificadas por Mickel y Smith (2004) y por lo tanto no consideradas para Tabasco por estos autores. Por lo anterior, considerando que había 118 especies y se aumentaron 21 de Cetzal-Ix et al. (2013a, b), suman 139 en total. Con las 30 nuevas adiciones de este estudio, se considera que la pteridoflora de Tabasco consta de 169 taxa (16.7\% de la riqueza de helechos de México). De igual forma aumentó a 54 el número de géneros para Tabasco, ya que previamente se conocían 46 (Mickel y Smith, 2004; Cetzal-Ix et al., 2013a, b).

De acuerdo con los datos anteriores, la zona de estudio contiene $37 \%$ de la riqueza de helechos y licopodios reportada para todo el estado en una fracción mínima de terreno $\left(0.9 \mathrm{~km}^{2}\right)$ comparada con la superficie total de Tabasco $\left(24,578 \mathrm{~km}^{2}\right)$. Este alto porcentaje presente en el sitio tiene sentido si se considera que la mayor superficie del estado se encuentra en terrenos por debajo de los 100 m s.n.m. (Díaz-Jiménez et al., 2015), donde la diversidad de especies de helechos es menor (Acebey et al., 2015; Carvajal-Hernández y Krömer, 2015), debido a que se presentan temperaturas más cálidas, baja disponibilidad de humedad ambiental y precipitaciones no constantes a lo largo del año (Kessler et al., 2011b), así como menor diferenciación de nichos ecológicos debido a una relativa homogeneidad paisajística (Kluge y Kessler, 2011). Dichos factores no son favorables para la sobrevivencia de los helechos. Por otro lado, las elevaciones presentes en la parte alta de la zona de estudio están cerca del rango altitudinal donde la diversidad de helechos aumenta hasta llegar al punto máximo (1000-2000 m s.n.m.) (Kluge et al., 2006; Salazar et al., 2015; Carvajal-Hernández et al., 2017).

Sin embargo, la altitud por sí misma no determina la diversidad de especies de helechos, si no que intervienen diferentes factores abióticos que se presentan con la altitud. Por ejemplo, las condiciones de temperaturas templadas y la constante entrada de humedad en la zona de estudio favorecen el establecimiento de especies de helechos que requieren condiciones de alta humedad y temperaturas templadas para el desarrollo del gametófito (Kessler, 2001). Contrario a las zonas bajas, la heterogeneidad ambiental que predomina en los hábitats montañosos favorece la presencia de una mayor cantidad de hábitats (efecto de las laderas y cañadas, rocas y árboles hospederos) que pueden ser ocupados por varias especies vegetales, incluyendo los helechos (Kessler et al., 2011b).

Las condiciones abióticas del sitio de estudio permiten el establecimiento del bosque mesófilo de montaña, el cual es uno de los ecosistemas terrestres con mayor diversidad vegetal del país (Rzedowski, 1991; Villaseñor, 2010). Además, se considera la taxocenosis de los helechos como uno de los elementos florísticos más conspicuos de este tipo de vegetación por la presencia y abundancia de individuos (Rzedowski, 1996; Williams-Linera et al., 2005; Carvajal-Hernández et al., 2014; Tejero-Díez et al., 2014). Por lo tanto, en la zona se combinan estos factores que favorecen la supervivencia de una gran diversidad de especies de helechos, incluyendo un alto porcentaje de epífitas (62\%), acorde al patrón de riqueza en un gradiente altitudinal (Salazar et al., 2015; Acebey et al., 2017a).

El bosque mesófilo de montaña de la zona ha sido poco explorado por la comunidad científica de México, ya que existen muy pocos estudios respecto a su riqueza florística o faunística (Gual-Díaz, 2014), o si existen, estos están sin publicar o dispersos en trabajos con temáticas distintas. Anteriormente la presencia de este tipo de bosque era desatendida en el estado, al grado de que no se consideraba su existencia en los diferentes estudios relacionados con la vegetación (López-Mendoza, 1980; Pérez et al., 2005; Villaseñor, 2010). Algunos de los trabajos que lo incluyen como un tipo de vegetación presente en Tabasco son la tesis no publicada de Almeida (2008) y recientemente el artículo de González-Aguilar y BureloRamos (2017), donde ambos mencionan una parte de la riqueza de orquídeas de la zona. Cabe señalar que, en la compilación de información sobre bosques mesófilos de México, editado por la Comisión Nacional para el Conocimiento y Uso de la Biodiversidad (CONABIO), se seña- 
la su presencia en Tabasco basada únicamente en el trabajo de Almeida (2008), sin corroborarlo con otros estudios debido a su escasez (Gual-Díaz y González-Medrano, 2014). Por lo tanto, el presente trabajo es probablemente el único estudio publicado hasta la fecha que aborda la diversidad florística que resguarda el fragmento de bosque mesófilo conocido en Tabasco, desde la perspectiva de los helechos y licopodios como grupos de estudio.

La presencia del bosque mesófilo de montaña en Tabasco se reduce a este único sitio; por lo tanto, no es extraño que la mayoría de los nuevos registros mencionados aquí provengan de este tipo de vegetación y hayan sido reportados previamente en los estados vecinos. La falta de estudios pteridológicos en la zona, y en el estado en general, se puede ejemplificar con el hecho de que especies de amplia distribución en México, no habían sido reportadas en los listados previos, tal es el caso de Asplenium cuspidatum Lam., Blechnum appendiculatum Willd., Parablechnum schiedeanum (C. Presl) Gasper \& Salino, Christella dentata (Forssk.) Brownsey \& Jermy, Hypolepis repens (L.) C. Presl, Pleopeltis angusta Humb. \& Bonpl. ex Willd. y Pteris quadriaurita Retz., incluso del género Elaphoglossum, el cual es uno de los más diversos del país y tampoco había sido reportado anteriormente en Tabasco.

Al hacer una comparación entre la riqueza y composición de helechos y licopodios en este sitio con respecto a otros bosques mesófilos a la misma altitud, se encuentran similitudes de riqueza, pero diferencias en composición, probablemente relacionadas con los factores abióticos. La riqueza reportada en el bosque mesófilo de montaña de este estudio (50 especies) es similar a la registrada en Los Tuxtlas (ubicado en el sureste de Veracruz) a esa misma altitud, en la cara noreste y suroeste del volcán de San Andrés Tuxtla (47 y 38 especies respectivamente) (Acebey et al., 2017b). Esta similitud en riqueza de especies puede estar relacionada con los valores de temperatura y precipitación igualmente similares en ambos sitios a dichas elevaciones (temperatura $=18 \mathrm{y}$ $20{ }^{\circ} \mathrm{C}$ y precipitación $=3000$ y $3500 \mathrm{~mm}$ ). Por otro lado, en el bosque mesófilo de montaña del municipio Yecuatla ubicado en la región centro del estado de Veracruz, se registran valores de riqueza similares (53 especies), aunque la temperatura promedio es más fría $\left(17^{\circ} \mathrm{C}\right)$ y la precipitación anual es menor (2000 mm). Si bien la riqueza es similar, por la ubicación geográfica del sitio del centro de Veracruz (a $40 \mathrm{~km}$ de la zona árida del Altiplano mexicano), la composición es distinta ya que se reportan géneros de afinidad Neártica (Mickel y Smith, 2004), por ejemplo, Polystichum Roth (tres especies) y Woodwardia Sm. (dos) que no se registraron en este estudio. Además, en Yecuatla existe poca representatividad de la familia Hymenophyllaceae (únicamente dos especies) y no se registraron helechos grammitidoides que requieren de humedad ambiental alta (Carvajal-Hernández et al., 2017). Por el contrario, en el bosque de Tabasco, se reportaron especies de afinidad tropical como Cyathea decurrentiloba Domin, Lomariopsis mexicana Holttum, seis especies de Hymenophyllaceae y tres del grupo de grammitidoides (Cuadro 2). Estos mismos grupos también fueron registrados en el Volcán de San Martín Tuxtla (Acebey et al., 2017b). Otra de las diferencias entre los sitios radica en la proporción de las especies epífitas, las cuales en este estudio constituyen $62 \%$ del total. En la zona de Los Tuxtlas, los helechos epifitos representan entre 70 y $80 \%$ de la riqueza total de especies (Acebey et al., 2017a) y en la zona centro de Veracruz, apenas 31\% (Carvajal-Hernández et al., 2014).

La diferente composición de especies y los valores contrastantes de porcentaje de epífitas del bosque del centro de Veracruz, con respecto a este estudio, demuestran la importancia de un clima húmedo para la distribución de los helechos. Además, corrobora la dificultad para encasillar al bosque mesófilo de montaña, bosque húmedo de montaña o bosque de niebla (como también es llamado) basados en la composición de especies o en parámetros climáticos (Villaseñor, 2010).

El reciente incremento de 51 nuevas adiciones al inventario de los helechos de Tabasco, 21 reportados por Cetzal-Ix et al. (2013a, b) y 30 en el presente estudio, manifiestan la necesidad de continuar con los inventarios florísticos regionales en el estado con énfasis en grupos taxonómicos como es el caso de los helechos. Además, 
estos grupos son importantes en cuanto a la aportación de la riqueza de especies en las floras locales en los trópicos, ya que representan entre 13 y $22 \%$ de la diversidad total estimada (Kelly et al., 1994; Kessler, 2010). Por lo tanto, los estudios florísticos que incluyan estos grupos estarán considerando un porcentaje importante de la diversidad local.

La zona representa un refugio de la biodiversidad de Tabasco, con mucho potencial para ser estudiado desde diferentes planteamientos científicos. Esto con la finalidad de incrementar el conocimiento sobre su biodiversidad y dinámica del ecosistema para sugerir medidas de conservación del único y restringido sitio con bosque mesófilo de montaña en Tabasco. Por otro lado, se requiere de un estudio mayor de la pteridoflora del estado que involucre trabajo en los herbarios y se complemente con exploraciones de campo, para obtener un listado actualizado de los helechos y licopodios y disminuir los vacíos de información que existen respecto a la diversidad de estos grupos.

\section{CONTRIBUCIÓN DE AUTORES}

$\mathrm{CICH}$ y LSM contribuyeron en el trabajo de campo. $\mathrm{CICH}$ identificó el material colectado. CICH, LSM, MK y ML participaron en la concepción y diseño del estudio, así como la redacción del manuscrito.

\section{FINANCIAMIENTO}

Este estudio fue apoyado por la Fundación Científica Alemana (DFG-Deutsche Forschungsgemeinschaft) en el Proyecto "Species richness patterns at the transition from the Tropics to the Subtropics: Studying environmental and historic effects" (LE1826/5-1).

\section{AgRADECIMIENTOS}

Los autores agradecen a Abiligam López-Velázquez, Pedro Díaz-Jiménez y Mauricio Juárez-Fragoso, por su colaboración en el trabajo de campo. A Samaria Armenta-Montero por la elaboración del mapa. Al personal del herbario CIB por el apoyo en el procesamiento de los ejemplares. A los dos revisores anónimos por las suge- rencias que ayudaron a enriquecer el manuscrito. Un especial agradecimiento para Alan Smith de la Universidad de California, Berkeley por su apoyo en la identificación y/o corroboración de las identidades de algunas especies aquí reportadas.

\section{LITERATURA CITADA}

Acebey, A. R., T. Krömer y M. Kessler. 2017a. Species richness and vertical distribution of ferns and lycophytes along an elevational gradient in Mexico. Flora 235: 83-91. DOI: https://dx.doi.org/10.1016/j.flora.2017.08.003

Acebey, A. R., T. Krömer, M. Vázquez-Torres y J. D. TejeroDíez. 2015. Helechos y licófitos de la Reserva de la Biosfera Los Tuxtlas, Veracruz, México. Botanical Sciences 93(2): 313-344. DOI: https://dx.doi.org/10.17129/botsci.124

Acebey, A. R., J. C. López-Acosta, J. D. Tejero-Díez y T. Krömer. 2017b. Riqueza y composición de helechos y licófitos en tres áreas de bosque mesófilo en los Tuxtlas, Veracruz, México. Revista Mexicana de Biodiversidad 88(3): 625635. DOI: https://doi.org/10.1016/j.rmb.2017.07.009

Almeida, C. C. M. 2008. Distribución espacial de la comunidad de orquídeas epífitas en la selva alta perennifolia y bosque mesófilo de montaña, en el ejido Villa Guadalupe, Huimanguillo, Tabasco, México. Tesis de licenciatura. Facultad de Ecología, Universidad de Juárez Autónoma de Tabasco. Villahermosa, México. 99 pp.

Arreguín-Sánchez, M. L., R. Fernández-Nava, R. Palacios-Chávez y D. L. Quiroz-García. 2001. Pteridoflora ilustrada del Estado de Querétaro. Instituto Politécnico Nacional, Secretaría de Educación Pública. México, D.F., México. 470 pp.

Bebber, D. P., M. A. Carine, J. R. I. Wood, A. H. Wortley, D. J. Harris, G. T. Prance, G. Davidse, J. Paige, T. D. Pennington, N. K. B. Robson y R. W. Scotland. 2010. Herbaria are a major frontier for species discovery. Proceedings of the National Academy of Sciences 107(51): 22169-22171. DOI: https://doi.org/10.1073/pnas.1011841108

Carvajal-Hernández, C. I. y T. Krömer. 2015. Riqueza y distribución de helechos y licófitos en el gradiente altitudinal del Cofre de Perote, centro de Veracruz, México. Botanical Sciences 93(3): 601-614. DOI: https:// dx.doi.org/10.17129/botsci.165 
Carvajal-Hernández, C. I., T. Krömer y M. Vázquez-Torres. 2014. Riqueza y composición florística de pteridobiontes en bosque mesófilo de montaña y ambientes asociados en el centro de Veracruz, México. Revista Mexicana de Biodiversidad 85(2): 491-501. DOI: https://doi. org $/ 10.7550 / \mathrm{rmb} .41292$

Carvajal-Hernández, C. I., T. Krömer, J. C. López-Acosta, J. A. Gómez-Díaz y M. Kessler. 2017. Conservation value of disturbed and secondary forests for ferns and lycophytes along an elevational gradient in Mexico. Applied Vegetation Science 20(4): 662-672. DOI: https://dx.doi. org/10.1111/avsc. 12318

Cetzal-Ix, W., E. Noguera-Savelli, M. Martínez-Icó y N. Ramírez-Marcial. 2013a. Diversidad de helechos y licófitos en fragmentos de selva mediana subperennifolia del sur de Tabasco, México. Botanical Sciences 91(3): 261-271. DOI: https://dx.doi.org/10.17129/botsci.7

Cetzal-Ix, W., E. Noguera-Savelli y N. Ramírez-Marcial. 2013b. Nuevos registros de helechos para Tabasco, México. Revista Mexicana de Biodiversidad 84(3): 977982. DOI: https://doi.org/10.7550/rmb.35605

Cowan, C. P. 1983. Listados Florísticos de México I. Flora de Tabasco. Instituto de Biología, Universidad Nacional Autónoma de México. México, D.F., México. 123 pp.

Díaz-Barriga, H. y M. Palacios-Ríos. 1992. Listado preliminar de especies de pteridófitas de los estados de Guanajuato, Michoacán y Querétaro. Flora del Bajío y de regiones adyacentes. Fascículo complementario III: 1-58.

Díaz-Jiménez, P., M. A. Guadarrama-Olivera y T. Croat. 2015. Diversidad florística de Araceae en el estado de Tabasco, México. Botanical Sciences 93(1): 131-142. DOI: https:// dx.doi.org/10.17129/botsci.238

Ebihara,A.,J.Y.Dubuisson,K.Iwatsuki,S.HennequinyM.Ito. 2006. A taxonomic revision of Hymenophyllaceae. Blumea 51(2): 221-280. DOI: https://doi.org/10.3767/000651906x622210

González-Aguilar, M. A. y C. M. Burelo-Ramos. 2017. Adiciones a la orquideoflora de Tabasco, México. Acta Botanica Mexicana 121: 161-167. DOI: https://dx.doi. org/10.21829/abm121.2017.1292

Gual-Díaz, M. 2014. Sistema de Información del bosque mesófilo de montaña en México: recopilación y sistematización de datos e información. In: Gual-Díaz, M. y A. Rendón-Correa (comps.). Bosques mesófilos de montaña de México: diversidad, ecología y manejo. Comisión Nacional para el Conocimiento y Uso de la Biodiversidad. México, D.F., México. Pp. 69-108.

Gual-Díaz, M. y F. González-Medrano. 2014. Los bosques mesófilos de montaña de México. In: Gual-Díaz, M. y A. Rendón-Correa (comps.). Bosques mesófilos de montaña de México: diversidad, ecología y manejo. Comisión Nacional para el Conocimiento y Uso de la Biodiversidad. México, D.F., México. Pp. 27-68.

INEGI. 1999. Perspectiva Estadística: Tabasco. Instituto Nacional de Estadística, Geografía e Informática. Aguascalientes, México. 106 pp.

Karger, D. N., O. Conrad, J. Böhner, T. Kawohl, H. Kreft, R. W. Soria-Auza, N. E. Zimmermann, H. P. Linder y M. Kessler. 2017. Climatologies at high resolution for the earth's land surface areas, Scientific Data 4, 170122. http://chelsa-climate.org/ (consultado octubre de 2017).

Kelly, D. L., E. V. J. Tanner, E. M. Lughadha y V. Kapos. 1994. Floristics and biogeography of a rain forest in the Venezuelan Andes. Journal of Biogeography 21(4): 421440. DOI: https://doi.org/10.2307/2845760

Kessler, M. 2001.Pteridophyte species richness in Andean forests in Bolivia. Biodiversity and Conservation 10(9): 14731495. DOI: https://doi.org/10.1023/a:1011811224595

Kessler, M. 2010. Biogeography of ferns. In: Mehltreter, K., L. R. Walker y J. M. Sharpe (eds.). Fern ecology. Cambridge University Press. New York, USA. Pp. 22-60. DOI: https://doi.org/10.1017/CBO9780511844898.003

Kessler, M., M. Moguel-Velázquez, M. Sundue y P. H. Labiak. 2011a. Alansmia, a new genus of grammitid ferns (Polypodiaceae) segregated from Terpsichore. Brittonia 63(2): 233-244.

Kessler, M., J. Kluge, A. Hemp y R. Ohlemüller. 2011b. A global comparative analysis of elevational species richness patterns of ferns. Global Ecology and Biogeography 20(6): 868-880. DOI: https://doi.org/10.1111/j.14668238.2011.00653.x

Kluge, J. y M. Kessler. 2011. Influence of niche characteristics and forest type on fern species richness, abundance and 
plant size along an elevational gradient in Costa Rica. Plant Ecology 212(7): 1109-1121. DOI: https://doi. org/10.1007/s11258-010-9891-x

Kluge, J., M. Kessler y R. R. Dunn. 2006. What drives elevational patterns of diversity? A test of geometric constraints, climate and species pool effects for pteridophytes on an elevational gradient in Costa Rica. Global Ecology and Biogeography 15(4): 358-371. DOI: https://doi.org/10.1111/j.1466-822x.2006.00223.x

Knobloch, I. W. y D. S. Correll. 1962. Ferns and fern allies of Chihuahua, Mexico. Texas Research Foundation Renner. Texas, USA. 198 pp.

Krömer, T., C. I. Carvajal-Hernández, A. R. Acebey y A. R. Smith. 2015. A decade of new pteridophyte records for the State of Veracruz, Mexico. American Fern Journal 105(3): 162-175. DOI: https://doi.org/10.1640/00028444-105.3.162

Lehnert, M. 2012. A synopsis of the species of Cyathea (Cyatheaceae-Polypodiopsida) with pinnate to pinnatepinnatifid frond. Phytotaxa 61(1): 17-36. DOI: https://doi. org/10.11646/phytotaxa.61.1.2

López-Mendoza, R. 1980. Tipos de vegetación y su distribución en el estado de Tabasco y norte de Chiapas. Universidad Autónoma de Chapingo. México, D.F., México. 122 pp.

Lot, A. y F. Chiang. 1986. Manual de Herbario. Administración y manejo de colecciones: Técnicas de recolección y preparación de ejemplares botánicos. Consejo Nacional de la Flora de México, A.C. México, D.F., México. 142 pp.

Magaña, P. y J. L. Villaseñor. 2002. La flora de México ¿Se podrá conocer completamente? Ciencias 66: 24-26.

Magaña-Alejandro, M. A. 1992. Helechos de Tabasco. Universidad Juárez Autónoma de Tabasco. Villahermosa, México. 273 pp.

Mendoza-Ruíz, A., J. Ceja-Romero y B. Pérez-García. 2016. Helechos y licófitos epífitos de Veracruz, México: riqueza y distribución. Acta Botanica Mexicana 114: 87-136. DOI: https://doi.org/10.21829/abm114.2016.1104

Mickel, J. T. 1992. Pteridophytes. In: McVaugh, R. (ed.). A descriptive account of the vascular plants of western Mexico Vol. 17. Flora Novo Galiciana. University of Michigan Press. Michigan, USA. Pp. 120-467.
Mickel, J. T. y J. M. Beitel. 1988. Pteridophyte flora of Oaxaca, Mexico. Memoirs of the New York Botanical Garden 46: $1-568$.

Mickel, J. T. y A. R. Smith. 2004. The pteridophytes of Mexico. Memoirs of the New York Botanical Garden 88: 1-1054.

Moran, R. C., P. H. Labiak y M. Sundue. 2010. Synopsis of Mickelia, a newly recognized genus of bolbitidoid ferns (Dryopteridaceae). Brittonia 62(4): 337-356. DOI: https:// doi.org/10.1007/s12228-010-9158-9

Oliveira, D. V. A., A. R. Smith y A. L. Gasper. 2017. Parablechnum roraimense and $P$. paucipinna spp. nov. (Blechnaceae: Polypodiopsida), lectotypification of $P$. stuebalii, and citation corrections in the family. Phytotaxa 292(1): 72-73. DOI: https://doi.org/10.11646/ phytotaxa.292.1.6

Palacios-Ríos, M. 1992. Las Pteridofitas del estado de Veracruz. Tesis de maestría. Facultad de Ciencias, Universidad Nacional Autónoma de México. México, D.F., México. 364 pp.

Pérez, L. A., S. M. Sousa, A. M. Hanan, F. Chiang y P. Tenorio. 2005. Vegetación terrestre. In: Bueno, J., F. Álvarez y S. Santiago (eds.). Biodiversidad del Estado de Tabasco. Instituto de Biología, Universidad Nacional Autónoma de México-Comisión Nacional para el Conocimiento y Uso de la Biodiversidad. México, D.F., México. Pp. 65-110.

PPG I (Pteridophyte Phylogeny Group I). 2016. A communityderived classification for extant lycophytes and ferns. Journal of Systematics and Evolution 54(6): 563-603. DOI: https://doi.org/10.1111/jse.12229

Ranker, T. A., A. R. Smith, B. S. Parris, J. M. O. Geiger, C. H. Haufler, S. C. K. Straub y H. Schneider. 2004. Phylogeny and evolution of grammitid ferns (Grammitidaceae): A case of rampant morphological homoplasy. Taxon 53(2): 415-428. DOI: https://doi.org/10.2307/4135619

Riba, R., L. Pacheco, A. Valdés y Y. Sandoval. 1996. Pteridoflora del estado de Morelos, México. Lista de familias, géneros y especies. Acta Botanica Mexicana 37: 45-65. DOI: https://dx.doi.org/10.21829/abm37.1996.769

Rojas-Alvarado, A. F. y J. D. Tejero-Díez. 2017. Novelties and notes in Lindsaea (Lindsaeaceae) from Mexico and Central America. Phytotaxa 296(2): 147-160. DOI: https://doi.org/10.11646/phytotaxa.296.2.4 
Rzedowski, J. 1991. Diversidad y orígenes de la flora fanerogámica de México. Acta Botanica Mexicana 14: 3-21. DOI: https://doi.org/10.21829/abm14.1991.611

Rzedowski, J. 1996. Análisis preliminar de la flora vascular de los bosques mesófilos de montaña de México. Acta Botanica Mexicana 35: 25-44. DOI: https://doi. org/10.21829/abm35.1996.955

Salazar, T. J. C. (ed.). 1994. Compendio monográfico de la historia de Tabasco: Obra literaria pedagógica. 2a ed. Villahermosa, México. 249 pp.

Salazar, L., J. Homeier, M. Kessler, S. Abrahamczyk, M. Lehnert, T. Krömer y J. Kluge. 2015. Diversity patterns of ferns along elevation in Andean tropical forests. Plant Ecology and Diversity 8(1): 13-24. DOI: https://doi.org/1 $0.1080 / 17550874.2013 .843036$

Sánchez-González, A., Y. Pérez-Atilano, A. G. HernándezÁlvarez, M. Gutiérrez-Lozano, M. A. Pérez-Paredes, E. Álvarez-Zuñiga y J. D. Tejero-Díez. 2017. Helechos y licopodios del estado de Hidalgo, México. In: RamírezBautista, A., A. Sánchez-González, G. Sánchez-Rojas y C. Cuevas-Cardona (eds.). Biodiversidad del estado de Hidalgo. Universidad Autónoma del Estado de HidalgoConsejo Nacional de Ciencia y Tecnología. Pachuca, México. Pp. 65-97.

Smith, A. R. 1981. Pteridophytes. Flora of Chiapas, Part 2. California Academy of Sciences. San Francisco, USA. $370 \mathrm{pp}$.

Smith, A. R. y J. D. Tejero-Díez. 2014. Pleopeltis (Polypodiaceae), a redefinition of the genus and new combinations. Botanical Sciences 92(1): 43-58. DOI: https://dx.doi. org/10.17129/botsci.29

Smith, A. R., K. M. Pryer, E. Schuettpelz, P. Korall, H. Schneider y P. G. Wolf. 2006. A classification for extant ferns. Taxon 55(3): 705-731. DOI: https://doi.org/10.2307/25065646
Sundue, M. A. 2017. Three new species of grammitid ferns (Polypodiaceae) from the fern hunter's paradise: Sierra Juárez, Oaxaca, Mexico. Systematic Botany 42(1): 160168. DOI: https://doi.org/10.1600/036364417X694962

Tejero-Díez, J. D. y M. L. Arreguín-Sánchez. 2004. Lista con anotaciones de los pteridófitos del estado de México, México. Acta Botanica Mexicana 69: 1-82. DOI: https:// doi.org/10.21829/abm69.2004.981

Tejero-Díez, J. D., A. Torres-Díaz y M. Gual-Díaz. 2014. Licopodios y helechos en el bosque mesófilo de montaña de México. In: Gual-Díaz, M. y A. Rendón-Correa (comps.). Bosques mesófilos de montaña de México: diversidad, ecología y manejo. Comisión Nacional para el Conocimiento y Uso de la Biodiversidad. México, D.F., México. Pp. 197-220.

Vergara-Rodríguez, D., G. Mathieu, M. S. Samain, S. ArmentaMontero y T. Krömer. 2017. Diversity, distribution and conservation status of Peperomia (Piperaceae) in the state of Veracruz, Mexico. Tropical Conservation Science 10: 1-28. DOI: https://doi.org/10.1177/1940082917702383

Villaseñor, J. L. 2010. El bosque húmedo de montaña en México y sus plantas vasculares: catálogo florístico taxonómico. Comisión Nacional para el Conocimiento y Uso de la Biodiversidad-Universidad Nacional Autónoma de México. México, D.F., México. 40 pp.

Villaseñor, J. L. 2016. Checklist of the native vascular plants of Mexico. Revista Mexicana de Biodiversidad 87(3): 559902. DOI: https://doi.org/10.1016/j.rmb.2016.06.017

Williams-Linera, G., M. Palacios-Ríos y R. Hernández-Gómez. 2005. Fern richness, tree species surrogacy, and fragment complementarity in a Mexican tropical montane cloud forest. Biodiversity and Conservation 14(1): 119-133. DOI: https://doi.org/10.1007/s10531-005-4053-5 